\title{
Formación en Diseño Industrial: una propuesta metodológica coherente con el desarrollo sostenible
}

Guillermo Andrés Alvarado Nieto', Paola Andrea Roa López ${ }^{2}$, Diana Lorena Zuleta Ortiz ${ }^{3}$

Recibido: 12-02-2016

Aceptado: 05-04-2016

\section{RESUMEN}

El presente artículo busca dar a conocer una propuesta metodológica para el diseño, producción, uso y desuso de artefactos con características compatibles con los principios del desarrollo sostenible. En este sentido, la metodología aplicada para su desarrollo se llevó a cabo dentro de los parámetros de investigación evaluativa cualitativa, descriptivo y aplicada, construida a partir de la identificación de criterios de sostenibilidad detectados en las metodologías de Diseño empleadas actualmente en la formación en Diseño Industrial para la configuración de artefactos, con la inclusión de otros criterios planteados a partir de elementos teóricos como la Producción más Limpia, el Eco Diseño, la teoría Cradle to Cradle, el Análisis de Ciclo de Vida, entre otros. Teniendo en cuenta lo anterior, los resultados nos indican que si se actúa en el método esto se evidencia en el resultado, logrando de esta forma, involucrar la gestión necesaria para obtener corrientes de salida principales y secundarias desde la comprensión del problema hasta la fase de uso y desuso que denoten responsabilidad con el medio ambiente y a la vez un vínculo estético con el usuario de manera que su permanencia en él evite ser desechado en corto tiempo.

1. Diseñador Industrial de la Universidad Jorge Tadeo Lozano, Bogotá. Magister en Desarrollo Sostenible y Medio Ambiente de la Universidad de Manizales, Docente, Investigador y Coordinador del Programa de Diseño Industrial de la Fundación Academia de Dibujo Profesional, Cali. Teléfono: 3002705619.

Correo: notas.academia@gmail.com.

2. Diseñadora Industrial de la Universidad Nacional de Colombia, sede Palmira. Magister en Desarrollo Sostenible y Medio Ambiente de la Universidad de Manizales, docente e investigadora del Programa de Diseño Industrial de la Fundación Academia de Dibujo Profesional, Cali. Teléfono: 3167502239.

Correo: paolaandrearoalopez@hotmail.com.

3. Ingeniera Industrial de la Pontificia Universidad Javeriana, Cali. Magister en Desarrollo Sostenible y Medio Ambiente de la Universidad de Manizales. Coordinadora de Proyectos del Centro de Consultoría y Educación Continua de la Pontificia Universidad Javeriana, Cali. Teléfono: 3006735212. Correo electrónico: dianazuor@gmail.com 
Palabras clave: Diseño Industrial, criterios de sostenibilidad, formación en diseño, métodos de configuración de artefactos o productos.

\title{
Training in Industrial Design: a consistent methodologicalapproachtosustainable development
}

\begin{abstract}
This paper seeks to present a methodological proposal for the design, production, use and disuse of artifacts with features that are compatible with sustainable development. In this sense, the methodology applied for their development was carried out within the parameters of qualitative, evaluative, descriptive and applied research, constructed based on the identification of criteria of sustainability detected in the current methodologies for teaching Industrial Design for the configuration of artifacts, as well as other criteria set forth based on the theoretical elements such as Cleaner Production, Eco Design, Cradle to CradleTheory, Life Cycle Analysis, among others. Considering this, the findings indicate that by using the method the results will be evident, obtaining in this way a complete understanding of the problem of obtaining principal and secondary solutions from the use stage to the disuse stage of an artifact which shows responsibility with the environment and simultaneously is an aesthetic link with the user so as to avoid discarding the artifact in the short term.
\end{abstract}

Key words: Education system, development, education, science, technology, innovation, curriculum.

\section{Introducción}

Hasta inicios del siglo XIX la ideación y la construcción de objetos fue desarrollada por una misma persona, el artesano, que ideaba sus productos y los elaboraba manualmente. Dichos objetos tenían características formales, cargadas de adornos que los convertían en piezas únicas de elevado valor que incrementaban el estatus de las personas que los adquirían. 
Los tiempos de producción y el costo de los objetos artesanales, llevaron a la aparición de la revolución industrial, que se dio en Inglaterra durante el periodo comprendido entre 1760 y 1830, en el cual se inició el reemplazo de la mano de obra con la mecanización y se instauró un nuevo sistema de producción. La producción industrial, hizo cambios al esquema artesanal y separó las tareas de concepción e ideación de las de fabricación o construcción, escisión que establece una etapa nueva en la división técnica del trabajo. El artesano generalmente configura y construye simultáneamente, mientras que el industrial se plantea un trabajo de pre concepción sistematizada, pues es impensable producir un objeto industrialmente sin antes haber definido minuciosamente sus características a fin de reducir las posibilidades de cambios que puedan generar un aumento en el costo, tiempo y trabajo. En la fase de concepción del objeto industrial es necesario definir aspectos funcionales, formales, tecnológicos, ergonómicos y psicológicos, entre otros.

Con la llegada de la Revolución Industrial la labor del diseñador industrial fue reconocida y apetecida en la sociedad, sin embargo, solo hasta 1969 el ICSID (International Council of Societies of Industrial Design) avala la definición de diseño industrial que en 1961 había propuesto el diseñador Tomás Maldonado:

"El Diseño Industrial es una actividad proyectual que consiste en determinar las propiedades formales de los objetos producidos industrialmente. Por propiedades formales no hay que entender tan solo las características exteriores, sino, sobre todo, las relaciones funcionales y estructurales que hacen que un objeto tenga una unidad coherente desde un punto de vista tanto del productor como del usuario, puesto que, mientras la preocupación exclusiva por los rasgos exteriores de un objeto determinado conlleva el deseo de hacerlo aparecer más atractivo o también disimular sus debilidades constitutivas, las propiedades formales de un objeto son siempre el resultado de la integración de factores diversos, tanto si son de tipo funcional, cultural, tecnológico o económico". (Maldonado, 1977)

La definición más actualizada propuesta por el ICSID es:

El diseño es una actividad creativa cuyo objetivo es establecer las cualidades multifacéticas de los objetos, procesos y servicios así como sus sistemas y sus ciclos de vida vitales de forma total. 
Por lo tanto, el diseño es el factor central para la innovación y la humanización de las tecnologías y un factor crucial para el intercambio cultural y económico .

En este sentido, el diseño industrial descubre las relaciones estructurales organizativas, funcionales, expresivas y económicas de la actividad de producir objetos con ética global que mejoren la sostenibilidad y protección del medio ambiente. Ética social, que permita beneficios y libertad humana, sea de manera individual o colectiva, velando por intereses de usuarios, productores y el mercado. Ética cultural, que vela por la cultura aunque sin dejar de lado la globalidad.

Adicionalmente, el diseño industrial se entiende como una profesión multidisciplinar que busca mejorar la calidad de vida de la sociedad con la satisfacción de necesidades objetuales que son producidas industrialmente (Roa, 2013).

En relación con la producción, la llegada de la revolución Industrial y el desarrollo del diseño industrial permitieron la fabricación industrial de los objetos, lo que permitió su repetición, y dio lugar a lo que hoy se conoce como producción en serie. Este sistema fue incorporado por primera vez porHenry Ford, yse convirtió en uno de los conceptos de productividad más poderosos de la historia y en gran medida responsable del surgimiento y la expansión del sistema industrializado basado en el consumo, existente en la actualidad. El auge de la producción industrial trajo ventajas para el desarrollo objetual de la sociedad pero también generó consecuencias negativas para el medio ambiente, dado que la producción masiva de objetos y la búsqueda de riquezas para la satisfacción de necesidades produjo problemas de contaminación, consumo de recursos naturales, pérdida de especies animales y vegetales, ecosistemas transformados, desplazamientos, etc., que afectan la calidad de vida de la sociedad en la actualidad y la de generaciones futuras.

Con el fin de avanzar en mecanismos de valoración de la degradación ambiental, ocasionada principalmente por el crecimiento económico de los países industrializados, se constituyó en el tema central de la Comisión Mundial sobre Medio Ambiente y Desarrollo en 1987, de la cual resultó el documento conocido como el informe Bruntland que incluyó la definición del concepto de desarrollo sostenible como el proceso de "Satisfacer las necesidades de las generaciones presentes sin comprometer las posibilidades de las del futuro para atender sus propias necesidades" 
(Comisión Mundial Sobre el Medio Ambiente y el Desarrollo, 1987). A pesar de la apropiación al parecer generalizada de esta definición, y de acuerdo con Goñi y Goin (2006) son muchos los interrogantes aún no resueltos. En este sentido y teniendo en cuenta que las necesidades humanas van más allá de las básicas y están relacionadas con múltiples dimensiones como la cultura, la política, el medio ambiente, la economía y la tecnología; surge la inquietud sobre si el límite de crecimiento y desarrollo se está definiendo a partir de la búsqueda de satisfacción de necesidades actuales y futuras cada vez más crecientes y exigentes de los seres humanos, o si por el contrario, el sistema biofísico es el que debe poner los límites a los patrones dominantes de producción y consumo (Leff, 2010).

Según Dalal-Clayton y Bass (2002), el desarrollo sostenible implica que las naciones sean capaces de lograr el desarrollo económico y social, sin degradar el ambiente a niveles irreversibles, de manera tal que se protejan los derechos y oportunidades de las generaciones venideras.

El desarrollo sostenible debe integrar tres componentes (Naciones Unidas, 2005): medioambiental, económico y social. Definiendo a su vez, desde sus interrelaciones, las condiciones necesarias para lograr escenarios que faciliten la generación de beneficios en los tres aspectos, ya que si se generan acciones a cualquier nivel que van en detrimento de alguno de los tres componentes sería una postura miope que ayudará a aumentar las brechas y estadios insostenibles en cualquiera de sus vértices.

En este sentido, en la búsqueda de materializar la aplicación de principios del desarrollo sostenible desde un enfoque preventivo en las empresas, se ha definido el modelo de ecología industrial que está orientado a "reducir el consumo de materias primas y energía hasta valores que la biosfera pueda reemplazar y que las emisiones y los residuos se reduzcan hasta valores que la biosfera pueda asimilar" (Capuz, y otros, 2004). La ecología industrial pertenece a un área del conocimiento que pretende que los sistemas industriales se comporten de manera similar a los ecosistemas naturales, transitando de un modelo de producción industrial lineal a uno cíclico. El modelo lineal no tiene en cuenta la huella de carbono generada en los procesos, las prácticas de extracción responsables, políticas laborales correctas, el post consumo, desconoce la perdurabilidad de los productos para darle movilidad al mercado, incentivando el consumo desmedido. Modelo contrario al cíclico, que toma en cuenta 
cuidadosamente cada decisión en el diseño y generación del producto con el fin de que al momento del desuso, pueda ir a la biósfera o ciclo biológico, es decir, que se integre de nuevo al ciclo medio ambiental, o se dirija a la tecnosfera o ciclo tecnológico en donde sus materias primas, componentes y tecnología, harán parte de una nueva cadena productiva (McDonough \& Braungart, 2005). De igual manera en cada una de las fases de generación del producto, se busca reducir el posible impacto ambiental impulsando de esta forma interacciones entre economía, el ambiente y la sociedad (Cervantes, Sosa, Rodríguez, \& Robles, 2009).

Ahora bien, al ser el diseño industrial un elemento crucial en esta cadena como el profesional que diseña y produce la cultura material, es de suma importancia asimilar estas teorías en torno a los procesos cíclicos como parte de un aporte a los métodos que rigen la configuración y desarrollo de productos en la disciplina del diseño industrial; puesto que su inclusión podría aportar al camino de transición hacia un producto sostenible. De igual manera y de acuerdo con Cervantes (2009), la importancia de los resultados que arroja la implementación de la ecología industrial, radica en que este enfoque ha logrado transformar los sistemas de producción lineales de diversas regiones en sistemas de ciclo cerrado donde todos los sectores que conforman la región se ven favorecidos. Y como lo sustenta también Cervantes (2009), la meta final de la ecología industrial es garantizar el desarrollo sustentable, bien sea en lo global, regional o local.

En el estudio o la implantación de un ecosistema industrial se pueden usar métodos y herramientas como la producción más limpia, el análisis de ciclo de vida, el análisis de flujo de materia, el análisis económicoambiental, la ecoeficiencia, los indicadores de desarrollo sostenible, las bolsas de residuos o subproductos, la huella de carbono y huella ecológica, análisis de redes sociales, etc. (Cervantes, Sosa, Rodríguez, \& Robles, 2009).

Para los alcances de esta investigación se tuvo en cuenta que los productos sostenibles son aquellos que desde su configuración estética, funcional y productiva involucran los principios del desarrollo sostenible y articulan elementos orientados por la ecología industrial, el análisis de ciclo de vida, el consumo sostenible, el ecodiseño cuyo objetivo final es la promoción del diseño de productos mejorados desde una perspectiva medioambiental (Bovea Edo, 2002) y la "ciclicidad" sustentada en la metodología de "Cradle to Cradle" (McDonough \& Braungart, 2005), que 
presenta un enfoque en el que los ciclos biológicos y técnicos se cierran sin efectos perjudiciales para el medio ambiente. En este enfoque, los materiales de desecho se convierten en "nutrientes" para un ciclo siguiente.

En la formación académica universitaria en diseño industrial es fundamental adquirir las bases estructurales del pensamiento sistémico en torno a la gestión y desarrollo de producto y estudiar los principales métodos es decir las diferentes etapas y procedimientos, técnicas, ayudas o herramientas para configurar artefactos y que se emplean en la formación de los estudiantes de diseño industrial, de manera que puedan articularse elementos del desarrollo sostenible en una ruta metodológica que contribuya al mejoramiento de las prácticas profesionales del mismo y con ello brindar un aporte al mejoramiento de los procesos industriales y empresariales de la región.

Los resultados de la investigación ofrecen elementos para crear una ruta metodológica en diseño, acorde al tipo de realidad social y ambiental en que vivimos; ser un constante motivador de la innovación que construya una cultura ambiental en la comunidad académica y profesional en diseño industrial a partir de la construcción de consciencia y capacidades en sintonía con el desarrollo sostenible,

En los contenidos académicos y programáticos de las diferentes asignaturas de diseño industrial, se identificaron seis métodos de diseño de artefactos usados para la formación académica, estos se tuvieron en cuenta para su correspondiente análisis y detección de criterios de sostenibilidad, con el fin de hacer de estos la base de configuración de la metodología de diseño ARZ, cuyo objetivo persigue el diseño, producción, uso y desuso de artefactos sostenibles:

1. Metodología de diseño propuesta por Ambrose-Harris (Ambrose \& Harris, 2010)

2. Metodología de diseño propuesta por Paul Rodgers (Rodgers

\& Milto, 2011)

3. Metodología proyectual, propuesta por Bruno Munari (Munari, 1983)

4. Metodología planteada por Gerardo Rodríguez Morales

(Rodríguez, 1996)

5. Métodos de diseño estrategia para el diseño de productos

(Cross, 2012) 
6. Guía metodológica de diseño industrial, propuesta por la Fundación Prodintec (Fundación Prodintec, 2013)

A continuación se presentan los criterios de sostenibilidad detectados en cada uno de los métodos de diseño:

\section{Metodología de diseño propuesta por Ambrose-Harris (Ambrose \& Harris, 2010)}

Gavin Ambrose es diseñador gráfico y trabaja para el sector de las artes, galerías, editores y agencias de publicidad. Es autor y diseñador de varios libros sobre identidad corporativa y embalaje de productos. Es licenciado en comunicación en la U. Central St. Martins de Londres, es editor y autor de varios libros relacionados con el ramo del diseño, la identidad corporativa y el embalaje de productos. Trabaja como diseñador autónomo.

Paul Harris es escritor y editor autónomo y colaborador en diversas revistas y periódicos. Se diplomó en London College Printing. Es autor de varias publicaciones relacionadas con el diseño de empaque.

La metodología de diseño expuesta por estos autores se enfoca en 7 fases: definición, investigación, ideación, prototipo, selección, implementación y aprendizaje.

\section{Criterios de sostenibilidad identificados en la metodología propuesta por Ambrose-Harris}

Dentro del proceso metodológico de los autores Ambrose Harris, no se evidencia con claridad una aproximación intencional a los elementos del desarrollo sostenible. La orientación de la ruta obedece más a los elementos del marketing que a una intención hacia el desarrollo social, económico o la protección del medio ambiente. Las recomendaciones que genera se enfocan principalmente al proceso creativo en el que desarrolla gran parte de su discurso. Por otro lado, no existe una definición clara de elementos de la producción o relaciones con el recurso humano. Sin embargo, se pueden detectar elementos de sostenibilidad presentes de manera secundaria en este método que enfocados debidamente en el plano del desarrollo sostenible, sirvan como insumo en el planteamiento 
de estrategias de desarrollo de producto. Es el caso de la etapa de definición en la que tanto el cliente como el equipo de diseño podrán establecer elementos o requerimientos claros que apunten al desarrollo económico y social, así como al bajo impacto ambiental. Esta definición de requerimientos no dependerá en sí de la metodología sino de la consciencia que en este ámbito las partes tengan frente al desarrollo del proyecto.

- De igual modo, el proceso deinvestigación dará pautas frente a los desarrollos existentes en línea con el producto que se está generando, de modo que sirvan como pauta y referente en el momento de realizar toma de decisiones.

- De la misma forma la etapa de ideación puede generar alternativas de producto que apunten al desarrollo sostenible, pero una vez más, no depende de la ruta metodológica en sí sino de las motivaciones del equipo creativo.

- Probablemente en la fase que mejor podría evidenciarse algún componente de criterios de sostenibilidad aplicados es en la fase de implementación en la que se habla de la elección de los materiales y en este punto cabe resaltar que los autores plantean el suceso de longevidad del producto a través de su correcta elección y esto se puede relacionar claramente con una intención de evitar su rápido desecho; lo que en términos de las problemáticas medioambientales significa un aporte en cuanto a evitar incluir desechos que los ecosistemas deban asimilar.

- Finalmente, podemos encontrar que cuando los autores hacen mención a una etapa final de aprendizaje, pueden presentarse elementos de análisis frente al impacto social, económico y ambiental que tras su implementación y uso se compilen de manera que tales resultados sean tomados como pauta de mejoramiento frente a las anteriores condiciones de desarrollo de producto.

\section{Metodología de diseño propuesta por Paul Rodgers y Alex Milton (Rodgers \& Milton, 2011)}

Paul Rodgers es en la actualidad referente en temas de diseño de producto dada la gran cantidad de investigaciones publicadas en libros, periódicos y conferencias. Ha participado como miembro regular en 
consejos editoriales de diseño en la Design Research Society(DRS), en la Engineering and Product Design Education (EPDE), la European Academy of Design (EAD), la International Association of Societies of Design Research (IASDR). Asimismo ha sido miembro del concejo editorial del periódico internacional, Design Studies y miembro del concejo editorial de Design Creativity and Innovation, entre otros. Desde el año de 2009 es profesor de filosofía del diseño en la North Umbria School of Design. Antes de ello trabajó durante los años de 1999 a 2009 en Edimburg Napier University. Asimismo trabajó en el Centro de Ingeniería y Diseño de la Cambridge University en investigación post-doctoral de 1996 a 1999, y como profesor titular de Diseño de Producto en Wolverhampthon University entre 1995 y 1996.

El proceso metodológico de diseño de producto de Paul Rodgers y Alex Milton propone tres fases principales: Investigación, antecedentes y etapa de exploración, de la cual se desprenden 14 sub fases para alcanzar el objetivo.

\section{Criterios de sostenibilidad identificados en la metodología propuesta por Paul Rodgers y Alex Milton}

Dentro del proceso metodológico de Paul Rodgers, se evidencian con claridad aproximaciones intencionales a los elementos del desarrollo sostenible. El autor plantea, en la etapa de Especificaciones de Diseño de Producto (PDS), por sus siglas en inglés, diferentes elementos a tomar en cuenta en la configuración total del producto y donde se abordan temas como:

- El tiempo de vida del producto, donde se busca medir el alcance frente al desuso del mismo

- Los materiales con los que se va a desarrollar, teniendo en cuenta su impacto medioambiental y retorno al sistema productivo

- Los aspectos políticos y sociales, como respuesta al estudio de grupo objetivo y usuario a través de pesquisas etnográficas - Las políticas de seguridad del mismo, así como su coherencia con los estándares del contexto, sea local o internacional, donde por lo general se establecen normatividades orientadas a proteger y posibilitar la calidad de vida a nivel ambiental y social 
- Por otra parte considera la importancia del reciclaje, facilidad de desmantelado y disposición final dentro de los procesos de fabricación.

\section{Metodología proyectual, propuesta por Bruno Munari (Munari, 1983)}

Bruno Munari es considerado "uno de los máximos protagonistas del Diseño Industrial y gráfico del siglo XX" ya que logró hacer contribuciones fundamentales en variados campos de la expresión visual como la escultura, el diseño industrial, el diseño gráfico y la cinematografía y en campos no visuales como la poesía, la didáctica y la escritura. Se interesó por la investigación en temas como el movimiento, la luz, y el desarrollo de la creatividad y la fantasía en la infancia mediante el juego.

Junto al especialista Lucio Fontana, "el perfectísimo" Bruno Munari domina la escena de arte milanés en las décadas de 1950 y 1960; es durante estos años que se gesta un boom económico en el que nace la figura del artista operador-visual que se convierte en asesor empresarial y que contribuye activamente en el renacimiento industrial italiano de la posguerra. Es además considerado uno de los protagonistas del arte programado y cinético, pero sobresale por la multiplicidad de sus actividades y por su grande e intensa creatividad en cada definición, en cada catalogación.

El método proyectual propuesto por Bruno Munari, se basa en los principios del método cartesiano y consiste en una serie de operaciones o fases necesarias, dispuestas en un orden lógico definido por lo que él denomina la experiencia. Propone trece fases de desarrollo: identificación del problema, definición del problema, descomposición del problema, recolección de datos, análisis de datos, creatividad, materiales y tecnología, experimentación, construcción del modelo demostrativo, verificación, dibujos constructivos, prototipaje y posible solución del problema.

\section{Criterios de sostenibilidad identificados en la metodología propuesta por Munari}

En cuanto a la inclusión de criterios de sostenibilidad, no se evidencia de manera explícita que oriente la inclusión de aspectos medioambientes, 
relacionados con las especificaciones a tener en cuenta para la selección de materiales y tecnología, tampoco involucra el análisis del uso y disposición final del objeto. Sin embargo, es importante resaltar los siguientes aspectos relacionados con los principios planteados por el desarrollo sostenible:

- En la fase de identificación del problema, se orienta que la solución planteada como respuesta a la necesidad analizada debe aportar al mejoramiento de la calidad de vida del usuario. Esto tiene directa relación con el componente social del desarrollo sostenible, el cual se ocupa de movilizar estrategias y acciones orientadas al logro de mejores condiciones de vida.

- Igualmente en la fase de identificación del problema, el autor hace relación a que "el diseñador no debe dejarse comprometer en una operación realizada únicamente en provecho de la industria y en perjuicio del consumidor", lo cual denota un llamado de atención frente al rol del diseñador para pensar en las necesidades y beneficios del consumidor y no en el rol de producción sin límite propuesto por la industria. Este tipo de observaciones del autor, aportan de algún modo a generar posiciones críticas en los diseñadores formados con esta metodología, y esto está relacionado directamente con los principios planteados por el desarrollo sostenible.

- Cinco de las doce fases de la metodología, están concentradas en el análisis y compresión del problema. Esto denota la importancia que le da el autor al entendimiento del problema, lo cual aumenta las posibilidades de poder generar soluciones reales a la necesidad y no respuestas superficiales que promuevan el lujo. Esto facilita que la relación usuario-artefacto sea más fuerte debido a que el usuario seguro valorará un objeto que responda adecuadamente a su necesidad y que además le proponga características adicionales que surgieron del análisis riguroso hecho por el diseñador de los diferentes componentes del problema. Esta relación usuario-artefacto posiblemente se traducirá en ciclos de vida más prolongados que retrasarán su disposición final.

- En la fase de experimentación, el autor orienta que la prueba de los materiales, de las técnicas y de los instrumentos, permite 
recoger informaciones sobre nuevos usos de un producto concebido para un único uso. Esto sin duda, está también relacionado con la posibilidad de alargar el ciclo de vida del artefacto, a partir de la búsqueda de usos secundarios, de manera tal que al cumplir su función principal pueda ser aprovechado en otras aplicaciones.

\section{Manual de Diseño Industrial planteado por Gerardo Rodríguez Morales (Rodríguez, 1996)}

Gerardo Rodríguez es mexicano y cuenta una amplia experiencia académica y trayectoria profesional en el área del diseño y de su implementación de más de 30 años. Director de la Escuela de Diseño; coordinador académico; presidente de comités dictaminadores; consejero académico; participante en comisiones docentes de trabajo para la elaboración de documentos fundamentales para las transformación de contenidos y ajustes al plan de estudios de diseño; jurado en concursos de diseño y en la Bienal Nacional de Diseño; autor de materiales y antologías didácticas; capacitador sensible dispuesto a asesorar alumnos o profesores.

Institucionalmente se le conoce con un alto sentido de responsabilidad, visión ética, crítico y generoso que ha transformado el pensamiento del quehacer del diseño por sus aportaciones didácticas y metodológicas en el campo. Autor de libros que han sido referencia obligada en la formación de diseñadores de todo el país.

Las fases de la metodología planteada por Gerardo Rodríguez Morales son seis principales: proyectar e investigar modalidades diferentes de intervención, problemas y problemática, tipología de problemas de diseño, planteamiento y estructuración del problema, proyectación o desarrollo del proyecto y producción o fabricación, de la cual se desprenden 25 sub fases más.

\section{Criterios de sostenibilidad identificados en la metodología propuesta por Gerardo Rodríguez:}

Gerardo Rodríguez en su método propone tres criterios que evaluados bajo las características sostenibilidad se convierten en herramientas para el logro de artefactos sostenibles, dado que el autor no hace 
referencia ellos como un recurso para la sostenibilidad de los productos a diseñar

- En la fase uno de proyectar e investigarmodalidades diferentes de intervención, Gerardo Rodríguez propone que proyectar es el primer paso del hombre para el control del ambiente. Es necesario indicar que aunque el autor en su recomendación ve el ambiente como el contexto o espacio en cual participará el artefacto a diseñar. Este ambiente evaluado a la luz de la sostenibilidad, permite la generación de reflexión al diseñador evidenciado la responsabilidad que se tiene al momento de diseñar un artefacto que respete los recursos naturales $y$ destacando que el proyectar es el primer paso para el control de las implicaciones al medio ambiente, objetivo perseguido por la sostenibilidad.

- Uno de los objetivos de la sostenibilidad es el desarrollo social por medio de la satisfacción de sus necesidades, objetivo que se evidencia en la fase de establecimiento del fenómeno o situación por analizar, cuando el autor sugiere observar las diversas variables en el medio y ver la posibilidad de intervención desde el diseño industrial como herramienta para servir a la sociedad.

- En la fase de ajuste definitivo del producto para su producción en serie, el autor propone tratar de optimizar el concepto de diseño simplificando y eliminando partes del producto, criterio que facilita la disminución de posibles corrientes de salidas negativas resultantes de operaciones productivas que no agregan un valor significativo al artefacto, estrategia de ecodiseño en la optimización de técnicas de producción y optimización funcional del producto.

\section{Estrategia para el diseño de productos propuesta por Nigel Cross (Cross, 2012)}

El británico Nigel Cross, es un profesor en estudios de diseño y pieza clave en la sociedad de investigación en diseño, especialmente en metodologías de diseño y el estudio de diseño de la cognición. Director del Departamento de Diseño e Innovación de la facultad de Tecnología en la Universidad Abierta del Reino Unido. Editor y jefe de la 
Revista Internacional de investigación y Estudios de Diseño, publicada trimestralmente por el Sevier Science, en colaboración con la Sociedad de Investigación de Diseño. Autor de diversos libros y artículos, entre los que se encuentran Can a Machine Design, The Automated Architect, Developments in Design Methodology, Engineering Design Methods, Designerly Ways of Knowing, Analysing Design Activity, Understanding how designers think and work.

Nigel Cross propone 7 fases para el diseño de productos: clarificación de objetivos, establecimiento de funciones, fijación de requerimientos, determinación de características, generación de alternativas, evaluación de alternativas y mejora de detalles.

\section{Criterios de sostenibilidad identificados en la metodología propuesta por Nigel Cross}

El autor Nigel Cross en su metodología Estrategia para el Diseño de Productos no evidencia o hace en énfasis en el desarrollo de criterios sostenibles, pero incluye criterios que desde la perspectiva de lo sostenible generan resultados favorables como son:

- En la fase de mejora de detalles Nigel Cross propone eliminar todo aquello que se pueda eliminar sin alterar la función, reducir o combinarse componentes, simplificar funcionamientos o ensambles, modificar los materiales o procesos productivos y estandarizar o modularizar partes o dimensiones, buscando la reducción de costos de fabricación. Es importante destacar que aunque el autor no plantea dicho criterio buscando resultados sostenibles sino reducción de costos. Sin duda alguna al aplicarlo en el desarrollo de un producto, se logrará un aporte significativo en la sostenibilidad desde la producción de dicho objeto, dado que eliminar componentes de un objeto o combinarlos, simplificar funcionamientos, estandarizar materiales y procesos productivos, aunque logra la reducción de costos del producto, también reduce el consumo de energías y recursos humanos y mecánicos, reduce el consumo de materia prima y potencializa el uso de la infraestructura actual de la empresa productora sin la adquisición de una nueva, disminuyendo así las posibles corrientes salidas negativas resultantes de operaciones productivas que no agregan un valor significativo al artefacto, 
logrando de esta manera un aporte significativo en la aplicación de estrategias de ecodiseño por medio de la optimización de técnicas de producción. De igual manera y evaluando el anterior criterio desde la sostenibilidad, el reducir el costo final de un producto potencializa la posibilidad de convertirse en un producto que se encuentra al alcance de más personas, siendo más elevada la cantidad que se ven beneficiadas positivamente con el uso del objeto diseñado, producido y vendido.

- Nigel Cross en su fase de mejora de detalles también destaca que otros aspectos a tener en cuenta para la calidad o valor de un producto son el garantizar la utilidad, confiabilidad, seguridad, ausencia de procesos complejos de mantenimiento, tiempo de vida largo, y que no genere o genere pocos subproductos desagradables o indeseables como ruido o calor. Es importante recalcar que aspectos como utilidad, confiabilidad, seguridad $y$ ausencia de proceso complejos de mantenimiento son características clave en el diseño de artefactos sostenibles, dado que posibilitan la aceptación y apropiación por parte de los posibles compradores y usuarios, permitiendo de esta forma que el artefacto tenga una vida larga en poder de sus destinatarios finales, lo que a su vez reduce la producción y consumo de otros artefactos y el desuso y desecho de los mismos.

Que el producto diseñado no genere o genere pocos subproductos desagradables o indeseables como ruido o calor, planteado por Nigel Cross en la fase anteriormente mencionada, es importante destacarla como una característica que permite alcanzar la sostenibilidad en el momento de uso del producto o artefacto, ya que estos son agentes que impactan negativamente el medio ambiente.

\section{Guía metodológica de diseño industrial, propuesta por la Fundación Prodintec (Fundación Prodintec, 2013)}

Esta guía metodológica, surge del Proyecto PREDICA (Promoción y estudio para un diseño industrial competitivo) el cual tuvo como objetivo, dar a conocer los beneficios del diseño y potenciar su incorporación en las PYMES del Principado de Asturias.

En la publicación de la metodología, incluyeron una guía con los casos 
prácticos del proyecto, donde quedaron recogidos los principios que giran alrededor de un proceso integral de diseño de productos, incluida su gestión, así como parámetros de valoración.

Las fases planteadas en la metodología son seis: diseño del concepto, diseño de detalle, oficina técnica e ingeniería de producto, producción, mercado y reciclaje.

\section{Criterios de sostenibilidad identificados en la metodología propuesta por Prodintec}

La ruta metodológica propuesta por Prodintec, aborda de manera explícita orientaciones, actividades y herramientas relacionadas con la gestión ambiental desde el diseño hasta el fin de uso del artefacto. Se identifica que el enfoque medioambiental es transversal a la metodología y se tiene en cuenta claramente en gran parte de la metodología.

- En la fase de definición estratégica orienta la importancia de determinar los aspectos ambientales del producto, a través de la utilización de herramientas como la Matriz MET, los EcoIndicadores, el software para el análisis de ciclo de vida, y la utilización de materias y componentes alternativos para evitar impactos medioambientales.

- En la fase de diseño de concepto incluye la realización del análisis de la relación producto-usuario, cuyo desarrollo riguroso se podrá traducir en atributos y características que fortalezcan este vínculo, de manera tal que el ciclo de vida del producto sea más largo a partir del deseo del usuario de no desprenderse de su objeto. En esta misma fase, como parte de las herramientas, se recomienda el uso de las estrategias de ecodiseño las cuales permitirán incluir explícitamente aspectos relacionados con selección de materiales de bajo impacto, optimización de las técnicas de producción y de los medios de distribución, la inclusión de atributos que permitan la reducción del impacto durante su uso y la optimización de la vida del producto.

- En la fase de ensayo y verificación, se recomienda la utilización de normativas que traten aspectos medioambientales (normas 
ISO 9001, ISO 14001) y dentro de las herramientas se orientan Eco-indicadores.

- En la fase de reciclaje y evaluación de impacto medioambiental, resaltan la importancia de tener en cuenta los materiales y el diseño para disminuir los impactos en el medio ambiente que se puedan desencadenar de un inadecuado manejo en la disposición final del producto, de manera tal que se propenda por la reutilización o el reciclaje.

La detección de los criterios de sostenibilidad en los métodos de diseño evaluados, al igual que la estructura de cada una de las metodologías han sido empleadas como base para la generación de la Ruta metodológica ARZ, empleada en el diseño de productos sostenibles.

\section{Propuesta de ruta metodológica - Método de diseño ARZ}

La ruta metodológica propuesta facilita al diseñador comprender una necesidad artefactual, para a partir de ello generar sus posibles soluciones, procesos de producción, definición de estrategias de mercadeo y venta, así como condiciones de uso y desuso. Este método articula los criterios identificados en los métodos existentes con la inclusión transversal de otros criterios de sostenibilidad.

La figura 1 representa las cinco fases propuestas en la ruta metodológica $A R Z$, con sus diferentes subfases y actividades. 


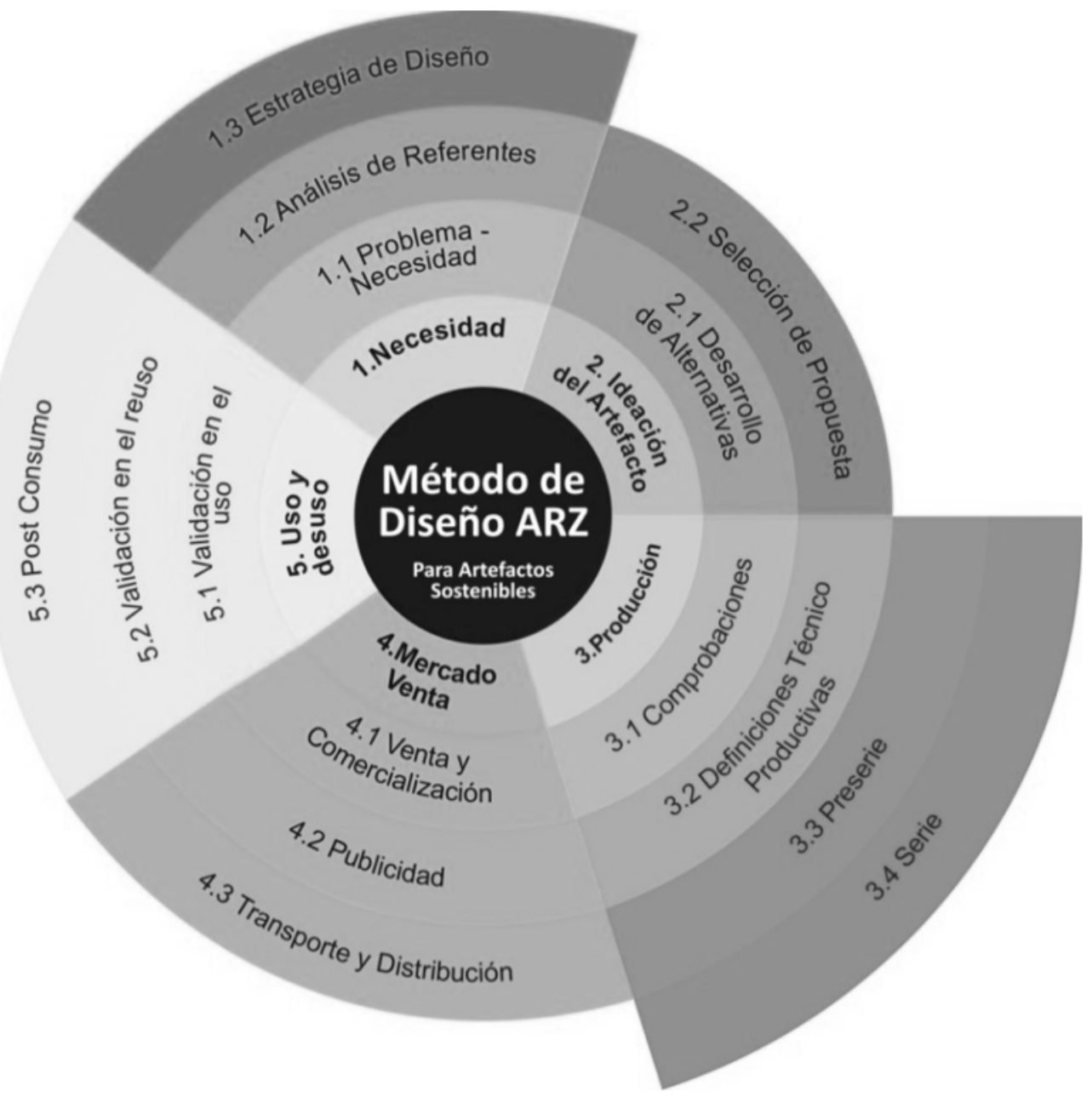

Figura 1. Método de diseño ARZ, para artefactos sostenibles.

\section{Criterios de sostenibilidad incluidos en el Método de diseño ARZ}

El diseño de la ruta metodológica ARZ es basado en los criterios detectados en los métodos evaluados anteriormente y en los criterios de sostenibilidad definidos a partir de los elementos articulados en el marco teórico del proyecto de investigación.

A continuación en la Tabla 1 se relacionan los criterios de sostenibilidad identificados en los métodos actualmente empleados con su correspondiente fase de aplicación en ARZ. 
Tabla 1. Criterios de Sostenibilidad detectados en las metodologías analizadas y su aplicación en ARZ

\begin{tabular}{|c|c|c|}
\hline Autor & Criterio sostenible propuesto & $\begin{array}{l}\text { Fase de aplicación } \\
\text { del criterio en ARZ }\end{array}$ \\
\hline \multirow{4}{*}{$\begin{array}{l}\text { Ambrose - } \\
\text { Harris }\end{array}$} & $\begin{array}{l}\text { Detectar en la investigación de referentes elementos } \\
\text { innovadores, que sirvan de bases a nuevo diseño. }\end{array}$ & Estado del arte \\
\hline & $\begin{array}{c}\text { Posibilidad de generar criterios sostenibles en la } \\
\text { ideación del producto. }\end{array}$ & $\begin{array}{c}\text { Aplicación } \\
\text { de modelos } \\
\text { sistémicos de } \\
\text { solución a los } \\
\text { requerimientos } \\
\text { planteados }\end{array}$ \\
\hline & $\begin{array}{l}\text { Elegir materiales que generen en el usuario el deseo } \\
\qquad \text { de conservar el producto }\end{array}$ & $\begin{array}{l}\text { Fijación de } \\
\text { requerimientos } \\
\text { de diseño }\end{array}$ \\
\hline & Retroalimentación del producto en sus fases de uso. & $\begin{array}{l}\text { Validación en } \\
\text { el uso }\end{array}$ \\
\hline \multirow{5}{*}{$\begin{array}{l}\text { Paul Rodgers y } \\
\text { Alex Milton }\end{array}$} & Proyección de la vida del producto. & $\begin{array}{l}\text { Fijación de } \\
\text { requerimientos } \\
\text { de diseño }\end{array}$ \\
\hline & $\begin{array}{l}\text { Seleccionar materiales teniendo en cuenta su impacto } \\
\text { medioambiental y retorno al sistema productivo. }\end{array}$ & $\begin{array}{l}\text { Fijación de } \\
\text { requerimientos } \\
\text { de diseño }\end{array}$ \\
\hline & $\begin{array}{l}\text { Comprender el grupo social objetivo dentro de su } \\
\text { contexto. }\end{array}$ & $\begin{array}{l}\text { Definición del } \\
\text { contexto de uso }\end{array}$ \\
\hline & Tener en cuenta la normatividad del contexto. & $\begin{array}{l}\text { Fijación de } \\
\text { requerimientos } \\
\text { de diseño }\end{array}$ \\
\hline & $\begin{array}{l}\text { Tener en cuenta en la fabricación el reciclaje, facilidad } \\
\text { de desmantelado y disposición final }\end{array}$ & $\begin{array}{l}\text { Fijación de } \\
\text { requerimientos } \\
\text { de diseño }\end{array}$ \\
\hline \multirow{4}{*}{ Bruno Munari } & $\begin{array}{l}\text { La solución planteada debe aportar al mejoramiento de } \\
\text { la calidad de vida del usuario }\end{array}$ & Necesidad \\
\hline & $\begin{array}{l}\text { El diseñador no debe dejarse comprometer en una } \\
\text { operación realizada únicamente en provecho de la } \\
\text { industria y en perjuicio del consumidor }\end{array}$ & $\begin{array}{l}\text { Definición de } \\
\text { la pertinencia } \\
\text { de solucionar } \\
\text { el problema } \\
\text { planteado }\end{array}$ \\
\hline & $\begin{array}{l}\text { Rigurosidad en el análisis y comprensión del problema } \\
\text { en busca de soluciones adecuadas. }\end{array}$ & $\begin{array}{l}\text { Comprensión } \\
\text { del problema y } \\
\text { definición de la } \\
\text { necesidad }\end{array}$ \\
\hline & $\begin{array}{l}\text { La prueba de los materiales, de las técnicas y de los } \\
\text { instrumentos, permite recoger informaciones sobre } \\
\text { nuevos usos de un producto concebido para un único } \\
\text { uso. }\end{array}$ & $\begin{array}{l}\text { Estado de la } \\
\text { técnica }\end{array}$ \\
\hline \multirow{3}{*}{$\begin{array}{l}\text { Gerardo } \\
\text { Rodríguez }\end{array}$} & $\begin{array}{l}\text { Proyectar es el primer paso del hombre para el control } \\
\qquad \text { del ambiente. }\end{array}$ & Necesidad \\
\hline & $\begin{array}{l}\text { La satisfacción de una necesidad como búsqueda del } \\
\text { desarrollo social }\end{array}$ & Necesidad \\
\hline & $\begin{array}{l}\text { Optimizar el concepto de diseño simplificando y } \\
\text { eliminando partes del producto }\end{array}$ & $\begin{array}{l}\text { Refinamiento de } \\
\text { la propuesta de } \\
\text { artefacto }\end{array}$ \\
\hline
\end{tabular}




\begin{tabular}{|c|c|c|}
\hline Autor & Criterio sostenible propuesto & $\begin{array}{l}\text { Fase de aplicación } \\
\text { del criterio en ARZ }\end{array}$ \\
\hline \multirow{2}{*}{ Nigel Cross } & $\begin{array}{l}\text { Eliminar todo aquello que se pueda eliminar sin } \\
\text { alterar la función, reducir o combinarse componentes, } \\
\text { simplificar funcionamientos o ensambles, modificar los } \\
\text { materiales o procesos productivos y estandarizar o } \\
\text { modular partes o dimensiones, buscando la reducción } \\
\text { de costos de fabricación. }\end{array}$ & $\begin{array}{l}\text { Refinamiento } \\
\text { de la propuesta } \\
\text { de artefacto }\end{array}$ \\
\hline & $\begin{array}{l}\text { Tener en cuenta para la calidad o valor de un producto } \\
\text { la utilidad, confiabilidad, seguridad, ausencia de } \\
\text { procesos complejos de mantenimiento, tiempo de vida } \\
\text { largo, y que no genere o genere pocos subproductos } \\
\text { desagradables o indeseables como ruido o calor. }\end{array}$ & $\begin{array}{l}\text { Fijación de } \\
\text { requerimientos } \\
\text { de diseño }\end{array}$ \\
\hline \multirow{4}{*}{$\begin{array}{l}\text { Fundación } \\
\text { Prodintec }\end{array}$} & $\begin{array}{c}\text { Uso de herramientas como la Matriz MET, los Eco- } \\
\text { Indicadores, el software para el análisis de ciclo } \\
\text { de vida, y la utilización de materias y componentes } \\
\text { alternativos. }\end{array}$ & $\begin{array}{l}\text { Fijación de } \\
\text { requerimientos } \\
\text { de diseño }\end{array}$ \\
\hline & Análisis de la relación producto-usuario & $\begin{array}{c}\text { Definición } \\
\text { del Usuario, } \\
\text { validación en el } \\
\text { uso, validación } \\
\text { de condiciones } \\
\text { de reuso }\end{array}$ \\
\hline & $\begin{array}{l}\text { Articulación de normativas que traten aspectos } \\
\text { medioambientales (normas ISO 9001, ISO 14001) y } \\
\text { herramientas Eco-indicadores. }\end{array}$ & $\begin{array}{l}\text { Fijación de } \\
\text { requerimientos } \\
\text { de diseño }\end{array}$ \\
\hline & $\begin{array}{l}\text { Tener en cuenta los materiales y el diseño para } \\
\text { disminuir los impactos en el medio ambiente }\end{array}$ & $\begin{array}{l}\text { Fijación de } \\
\text { requerimientos } \\
\text { de diseño }\end{array}$ \\
\hline
\end{tabular}

Fuente. Producción propia.

Para el desarrollo de la ruta metodológica ARZ, además de los criterios anteriormente relacionados, se tuvieron en cuenta otros criterios definidos a partir de la investigación desarrollada teniendo como base el marco teórico. Con la articulación de estos nuevos criterios compilados en la Tabla 2 con los ya identificados, se buscó obtener una ruta metodológica con mayores posibilidades de obtención de artefactos sostenibles. 
Tabla 2. Otros criterios de sostenibilidad incluidos en el método de diseño ARZ

\begin{tabular}{|c|c|}
\hline FASE & CRITERIO \\
\hline $\begin{array}{l}\text { Definición de la pertinencia } \\
\text { de solucionar el problema } \\
\text { planteado }\end{array}$ & Ética del diseñador al momento de abordar un proyecto de diseño. \\
\hline \multirow{4}{*}{$\begin{array}{l}\text { Fijación de requerimientos } \\
\text { de diseño }\end{array}$} & Tener en cuenta los aspectos ambientales de eco diseño \\
\hline & $\begin{array}{c}\text { Proyectar la optimización de la vida útil del producto buscando generar } \\
\text { confiabilidad y durabilidad, fácil mantenimiento y reparación, y un diseño clásico } \\
\text { que fortalezca la relación usuario-producto }\end{array}$ \\
\hline & $\begin{array}{l}\text { La fácil recuperación o reciclado de materiales, su fácil desmantelamiento y su } \\
\text { incineración segura o bien, su retorno a la biósfera (biodegradabilidad) con bajo } \\
\text { impacto }\end{array}$ \\
\hline & $\begin{array}{l}\text { Optimización de sus sistemas de empaque y comercialización, así como una } \\
\text { logística energéticamente eficiente en su distribución. }\end{array}$ \\
\hline \multirow{2}{*}{$\begin{array}{l}\text { Aplicación de modelos } \\
\text { sistémicos de solución a los } \\
\text { requerimientos planteados }\end{array}$} & $\begin{array}{l}\text { Incluir en la lluvia de ideas posibles soluciones de ámbito sostenible } \\
\text { (ambientales, económicas o sociales) }\end{array}$ \\
\hline & $\begin{array}{c}\text { Aplicación de preguntas filtro para destacar opciones amigables con el medio } \\
\text { ambiente, la sociedad y los factores económicos. }\end{array}$ \\
\hline $\begin{array}{l}\text { Realización de bocetación } \\
\text { o modelado de alternativas } \\
\text { de diseño }\end{array}$ & $\begin{array}{l}\text { Tener en cuenta reducir la impresión indiscriminada y el uso de papel excesivo y } \\
\text { buscar el uso de recursos de bocetación amigables con el medio ambiente. }\end{array}$ \\
\hline $\begin{array}{l}\text { Definición de corrientes de } \\
\text { entrada y salida }\end{array}$ & $\begin{array}{l}\text { Desarrollo con base en el formato de Identificación y registro de corrientes de } \\
\text { entrada }\end{array}$ \\
\hline \multirow{3}{*}{$\begin{array}{l}\text { Identificación y aplicación } \\
\text { de estrategias de gestión } \\
\text { ambiental en la planeación } \\
\text { técnico-productiva }\end{array}$} & $\begin{array}{l}\text { Las corrientes de salida deben ser analizadas y tratadas de manera que puedan } \\
\text { tomar la ruta de la biósfera o ciclo biológico, es decir, que se integren de nuevo } \\
\text { al ciclo medio ambiental sin producir impactos importantes. }\end{array}$ \\
\hline & Análisis tipo de materia prima o insumos y posibilidades de corrientes de salida. \\
\hline & $\begin{array}{l}\text { Desarrollo de manuales de usuario, con la ayuda de los planos de preserie } \\
\text { que den a conocer el desarmado o desensamblado del artefacto, bien sea } \\
\text { para orientar usos posteriores o secundarios o la apropiada separación de los } \\
\text { componentes de manera que sean adecuadamente dispuestos en la biosfera o } \\
\text { tecnosfera. }\end{array}$ \\
\hline Publicidad & Desarrollo de la matriz objetivos publicitarios según la fase del ciclo de vida. \\
\hline \multirow{3}{*}{ Transporte y distribución } & $\begin{array}{l}\text { Definir el medio de transporte que mejor garantice el cumplimiento de tiempos } \\
\text { de entrega al cliente o usuario con los menores impactos ambientales posibles. }\end{array}$ \\
\hline & Plantear opciones de transporte alternativo y eficiente. \\
\hline & Análisis de las condiciones de uso del artefacto. \\
\hline $\begin{array}{l}\text { Validación de condiciones } \\
\text { de reuso }\end{array}$ & $\begin{array}{l}\text { Validación y evaluación de los comportamientos relacionadas en primer caso } \\
\text { con las condiciones de uso planificadas, en donde se verificará si lo planeado es } \\
\text { en verdad una realidad en el usuario dentro de su contexto específico. }\end{array}$ \\
\hline $\begin{array}{l}\text { Validación de condiciones } \\
\text { de reuso }\end{array}$ & $\begin{array}{c}\text { Análisis de las funciones no planificadas del objeto, para retroalimentar los } \\
\text { procesos de relanzamiento de producto de manera que brinde al usuario } \\
\text { diferentes posibilidades configurativas de reuso. }\end{array}$ \\
\hline
\end{tabular}




\begin{tabular}{|c|c|}
\hline FASE & CRITERIO \\
\hline Postconsumo & $\begin{array}{c}\text { Inclusión dentro de la configuración de sus productos, la condición de posibilitar } \\
\text { su inclusión en el ciclo de la tecnosfera mediante el reciclaje, o de lo contrario su } \\
\text { correcto ingreso al ciclo de la biósfera, de acuerdo con su tiempo de vida. }\end{array}$ \\
\hline Postconsumo & $\begin{array}{c}\text { El producto deberá poseer características estéticas que dirijan la mirada del } \\
\text { usuario a la consciencia del post consumo y asimismo, el diseñador facilitará } \\
\text { mediante estrategias de medios, alianzas con el sector productivo etc., la } \\
\text { recolección y adecuada disposición del producto una vez finalizada su vida útil. }\end{array}$ \\
\hline $\begin{array}{c}\text { No se debe como diseñador delegar la responsabilidad única de la disposición } \\
\text { final al usuario y desviar la mirada frente a una responsabilidad inherente a } \\
\text { la profesión. Por lo anterior, será un requerimiento indispensable dentro de } \\
\text { la configuración del producto, el situar a este encada momento de su vida, } \\
\text { teniendo siempre presente, la ciclicidad en su desarrollo. }\end{array}$ \\
\hline
\end{tabular}

Fuente. Producción propia.

La inclusión de criterios de sostenibilidad fue trasversal a la ruta metodológica ARZ, con una mayor y estratégica aplicación de criterios en fases como fijación de requerimientos, técnico productiva, transporte, distribución, condiciones de reuso y postconsumo; dado que son fases en las cuales la planeación y desarrollo se convierten en estrategia de la inclusión de criterios que permitan el diseño, producción, comercialización y postconsumo de artefactos sostenibles. En la ruta metodológica ARZ son contemplados los 22 criterios evidenciados en los métodos actualmente utilizados más 23 criterios adicionales. En total los 45 aspectos abarcados por ARZ generan mayores potencialidades de obtener artefactos con criterios sostenibles más evidentes y significativos, contribuyendo de esta forma a la contaminación ambiental generada actualmente en el diseño y producción de artefactos.

De acuerdo al análisis de los métodos de diseño empleados en la formación de la carrera de técnico profesional en producción en diseño industrial, podemos señalar que los métodos planteados por la Fundación Prodintec, y Paul Rodgers \& Alex Milton, son los que más contemplan criterios de Desarrollo Sostenible para la configuración de producto, al abordar aspectos relevantes en las fases relacionadas con el planteamiento de requerimientos de diseño, la producción y el postconsumo. En contraste a ello, el método planteado por los teóricos Ambrose \& Harris, no establece parámetros específicos en este aspecto, sino que sugiere una ruta que depende de criterios particulares del diseñador en cuanto a este ámbito, más que del método en sí. De otro lado, métodos como los de Bruno Munari, Nigel Cross y Gerardo Rodríguez, proponen sin una clara intención frente al Desarrollo Sostenible, acciones que a la luz de este enfoque, pueden ser tomados en cuenta para encaminar las acciones que propone el método hacia la constitución de productos sostenibles. 
La inclusión de criterios metodológicos en torno a la sostenibilidad probablemente obedece también, al momento histórico en que cada una de ellas fue desarrollada. Se puede señalar cómo a nivel cronológico, existe una relación en donde las más recientes poseen mayores características mientras que las menos recientes se alejan de tales pautas.

Como parte de la creación de la ruta metodológica ARZ propuesta por los autores, fue necesario tomar en cuenta para su realización, una visión interdisciplinaria que abarcara la totalidad de sus planteamientos, desde la génesis del problema hasta la gestión de la disposición final del producto. Para ello, debieron tomarse en cuenta no solamente valores encaminados al desarrollo sostenible sino también elementos que fuesen de utilidad dentro de las estructuras metodológicas aportadas por cada uno de los teóricos analizados. Lo anterior permitió desarrollar la ruta metodológica ARZ en la que fueron contemplados los 22 criterios evidenciados en los métodos actualmente utilizados más 23 criterios adicionales. Se concluye entonces, que los 45 criterios de sostenibilidad abarcados por ARZ, dado lo holístico de su abordaje, generan mayores potencialidades de obtener artefactos con criterios sostenibles más evidentes y significativos.

Los resultados de este estudio nos plantean como docentes, la necesidad de formación en sostenibilidad para acompañar con mayor eficiencia y rigurosidad los procesos de desarrollo de producto, así como creadores de conciencia en el consumo y disposición final responsables. Adicionalmente a ello, se debe hacer la reflexión de si las actuales políticas de formación en infancia y juventud son consecuentes con dicho rumbo. Como formadores y ponentes de este estudio consideramos que este ejercicio, posibilita la articulación de estas facetas. 


\section{Referencias bibliográficas}

Ambrose, G., \& Harris, P. (2010). Metodología del Diseño. Barcelona, Parramón.

Bovea Edo, M. D. (2002). Valoración de Productos Ecológicos, aplicación al Diseño de Mobiliario de Oficina. Castellón, Universitat Jaume I.

Capuz, S., Gómez, T., Vivancos, J., Viñoles, R., Ferre, P., \& López, R. (2004). Ecodiseño: Ingeniería del ciclo de vida para el desarrollo de productos sotenibles. México D.F, Alfaomega grupo editor.

Cervantes, Sosa, Rodríguez, \& Robles. (2009). Ecolología Industrial y Desarrollo Sustentable. Ingeniería, Revista Académica de la FI-UADY, 13-1, 63-70.

Comisión Mundial Sobre el Medio Ambiente y el Desarrollo. (1987). Informe Brundtland. ONU.

Cross, N. (2012). Métodos de Diseño. Estrategias para el Diseño de Productos. Mexico, Limusa Wiley.

Dalal-Clayton, D., \& Bass, S. (2002). Sustainable Development strategies: A Resource Book. Londres, Earthscan Publications Ltd.

Ecodiseño. Diseño responsable de competitividad. (5 de 11 de 2010). Obtenido de http:// www.xn--ecodiseo-j3a.org/

Fundación Forum Ambiental. (2003). Forum ambiental. Obtenido de www.forumambiental. org/pdf/guiacast.pdf

Fundación PRODINTEC. (2013). Guía Metodológica de Diseño Industrial. Asturias, Prodintec.

Goñi, R., \& Goin, F. (2006). Marco Conceptual para la definición del desarrollo sustentable. Red Salud Colectiva, 191-198.

Ludevid, M. (2000). La gestión ambiental de la empresa. Barcelona, Ariel.

Maldonado, T. (1977). El Diseño Industrial Reconsiderado. Barcelona, Gustavi Gili.

McDonough, W., \& Braungart, M. (2005). Cradle to cradle (de la cuna a la cuna): rediseñando la forma en que hacemos las cosas. Madrid, McGraw-Hill.

Munari, B. (1983). ¿Cómo nacen los objetos?, apuntes para una metodología proyectual. Barcelona, Gustavo Gili.

Naciones Unidas. (24 de Octubre de 2005). Documento Final de la Cumbre Mundial 2005. Recuperado el 15 de 07 de 2014, de Documento Final de la Cumbre Mundial 2005: http:// daccess-dds-ny.un.org/doc/UNDOC/GEN/N05/487/63/PDF/N0548763.pdf?OpenElement 
Programa de las naciones unidas, unidad para el medio ambiente y la industria. (1999). Producción más limpia. Obtenido de www.pnuma.org/industria/publicaciones.php

Rodgers, P., \& Milton, A. (2011). Diseño de Producto. Barcelona, Promopress.

Rodríguez, G. (1996). Manual del Diseño Industrial. Mexico, Gustavo Gili.

Sanes Orrego, A. (2012). El Análisis de Ciclo de Vida en el Desarrollo Sostenible: Propuesta Metodológica para la Evaluaión de la Sostenibilidad de Sistemas Productivos. Bogotá, Universidad Nacional de Colombia. 